\title{
Long-term variability in the solar diurnal tide observed by HRDI and simulated by the GSWM
}

\author{
M. D. Burrage, ${ }^{1}$ M. E. Hagan, ${ }^{2}$ W. R. Skinner, ${ }^{1}$ D. L. Wu, ${ }^{3}$ and P. B. Hays ${ }^{1}$
}

\begin{abstract}
Observations of the mesosphere and lower thermosphere winds obtained by the High Resolution Doppler Imager (HRDI) on the Upper Atmosphere Research Satellite (UARS) during 1991 to 1995 reveal a semiannual variation in the amplitude of the $(1,1)$ diumal tide. The global-scale wave model (GSWM) represents the first numerical modeling attempt at simulating this seasonal variability, and a preliminary comparison of the GSWM tidal results with HRDI measurements is presented. The results of the comparison and of numerical tests point to some vital and unresolved questions regarding tidal dissipation and tropospheric forcing. In addition to the seasonal variability, HRDI has revealed a strong interannual modulation of the diurnal tide with amplitudes observed to change by nearly a factor of 2 from 1992 to 1994.
\end{abstract}

\section{Introduction}

The dominant global-scale wave in the low-latitude mesosphere and lower thermosphere (MLT) is the $(1,1)$ diurnal migrating tide generated primarily by solar heating in the troposphere and propagating into the middle atmosphere. Three-dimensional numerical simulations by general circulation models [e.g., Fesen et al., 1986] are unsuited to the study of long-term tidal variability due to their complexity and consequent computational requirements. Most theoretical treatments of solar tides employ two-dimensional linearized equations, and an approach derived from classical tidal theory [Chapman and Lindzen, 1970]. Improvements over the classical theory include the addition of the effects due to molecular and eddy diffusion, differential cooling, electrodynamic forces, and background winds and temperature gradients. These advances have been reviewed by Vial and Forbes [1989].

By taking advantage of updated heating rates [Groves, 1982a, b] and improved eddy diffusivity parameterizations [Garcia and Solomon, 1985], Forbes and Vial [1989] were able to simulate the seasonal variability of the semidiurnal tide, and Manson et al. [1989] found these predictions to be in reasonable agreement with radar observations. However, until very recently there has been no attempt to reproduce the strong seasonal variability of the diurnal tide which has been detected both from the ground [e.g., Vincent et al., 1989] and from space [Hays et al., 1994]. One of the aims of the latest version of the global-scale wave model (GSWM), developed by Hagan et al. [1995], is to address this type of tidal variability. The numerical framework of the GSWM is based upon the model by Forbes [1982], but incorporates several new features. Improvements to the background at-

\footnotetext{
${ }^{1}$ Space Physics Research Laboratory, University of Michigan, Ann Arbor

${ }^{2}$ National Center for Atmospheric Research, High Altitude Observatory, Boulder, CO

${ }^{3}$ Jet Propulsion Laboratory, California Institute of Technology, Pasadena
}

Copyright 1995 by the American Geophysical Union.

Paper number 95GL02635

0094-8534/95/95GL-02635\$03.00 mosphere used have been described by Hagan et al. [1993]. In addition, the GSWM employs the Groves [1982b] formulae for water vapor heating rates in the troposphere, providing the currently best available information on the tropospheric tidal forcing, which is the main cause of the diurnal tide in the MLT region. The influence of gravity waves on tidal dissipation is now included in the model, and the parameterization of this effect is detailed in Hagan et al. [1995].

The High Resolution Doppler Imager (HRDI) [Hays et al., 1993] on the Upper Atmosphere Research Satellite (UARS) has provided wind measurements in the mesosphere and lower thermosphere (MLT) since November 1991, permitting seasonal and interannual changes to be addressed. The HRDI technique, which brings a global perspective to the observation of MLT tidal dynamics, is complementary to the very detailed but localized radar data sets currently available. Since the tides are a global phenomena, the handful of localized stations providing MLT wind measurements do not provide a comprehensive test of numerical models. The present study serves to assess the current understanding of the diurnal tide, which is in turn critical to the understanding of the dynamics of the upper mesosphere and lower thermosphere. The approach involves 1) evaluation of GSWM predictions of the seasonal variability of the diurnal tide by comparison with HRDI observations, and 2) using the results of numerical experiments with this new model to point to the physical processes governing the observed behavior.

\section{HRDI Observations}

The inclination of the UARS orbit is $57^{\circ}$ and the HRDI telescope views a parallel strip at a latitudinal distance of about $15^{\circ}$ from the orbital track. Consequently, the maximum latitude observed is $72^{\circ}$ in one hemisphere and $42^{\circ}$ in the other hemisphere. Over the course of a 24 -hour period, HRDI obtains complete longitudinal coverage. During the daytime, measurements are collected over the altitude range $50-115 \mathrm{~km}$, while at night, a relatively narrow $\mathrm{O}_{2}$ emission layer is observed, effectively restricting the HRDI wind data to a single altitude near $95 \mathrm{~km}$ [Burrage et al., 1994]. On a given day the local time of the observations at a fixed latitude is nearly constant. A slow precession in the UARS orbit of $5^{\circ}$ in longitude (corresponding to 20 minutes in local time) per day provides a coverage of all local times in about 36 days. Thus, HRDI is able to readily detect coherent local time effects, such as the migrating diurnal and semidiurnal tides, by combining data collected over several weeks. In addition, the accumulation of over 3 years of data facilitates the study of seasonal variability.

Despite the local time restriction of the HRDI sampling on a single day, it is possible to obtain daily estimates of the diurnal tidal amplitude and phase. This is because monthly mean profiles of the diurnal tide are relatively stable in latitude and altitude since they are dominated by the tidal mode corresponding to the $(1,1)$ Hough function. To avoid significant contamination from mean winds and the mesosphere semiannual oscillation (MSAO), the analysis is limited to the meridional wind component. The technique has been described by Hays et al. [1994], and only a summary is given here. First monthly composited winds are formed providing coverage in altitude, latitude, and 12 hours of local time. By fitting the composite meridional winds to 
a 24-hour harmonic function and the $(1,1)$ expansion function, the altitude growth envelope and the vertical wavelength of the tide is established. The second step involves fitting daily measurements to a model of the following form:

$$
\begin{aligned}
v_{i j}= & \zeta\left(z_{j}\right) V\left(\theta_{i}\right)\left\{A \cos \left[2 \pi\left(\frac{z_{j}}{\lambda_{2}}+\frac{t_{i}}{24 h}\right)\right]\right. \\
& \left.+B \sin \left[2 \pi\left(\frac{z_{j}}{\lambda_{z}}+\frac{t_{l}}{24 h}\right)\right]\right\},
\end{aligned}
$$

where $\zeta$ is the growth envelope, $V$ is the meridional expansion function, $\theta$ is latitude, $z$ is altitude, $\lambda$ is vertical wavelength and $t$ is local time. The indices $i$ and $j$ denote the position along the measurement track and the altitude, respectively. There are only two free parameters in equation (1), $A$ and $B$, which yield the amplitude $\left(\sqrt{A^{2}+B^{2}}\right)$ and phase $\left(\tan ^{-1}(B / A)\right)$. In the first step only 12 hours of local time are fitted since nighttime measurements are not available (except for one altitude). This incomplete coverage yields a poorer fit than for 24-hour sampling. Since the diurnal tide dominates the lower latitude region, in the daily fitting a latitude restriction of $\pm 35^{\circ}$ is specified so as to increase the quality of the Hough mode analysis. The vertical wavelength has been found to be remarkably constant with time in the HRDI data set, with the best fits yielding a value of $25 \mathrm{~km}$ below an altitude of $90 \mathrm{~km}$ and $23 \mathrm{~km}$ above this altitude. These values for $\lambda_{z}$ were therefore fixed in the daily fitting analysis. For the altitude of $95 \mathrm{~km}$, where HRDI also obtains nighttime measurements, a more orthodox analysis would be possible.
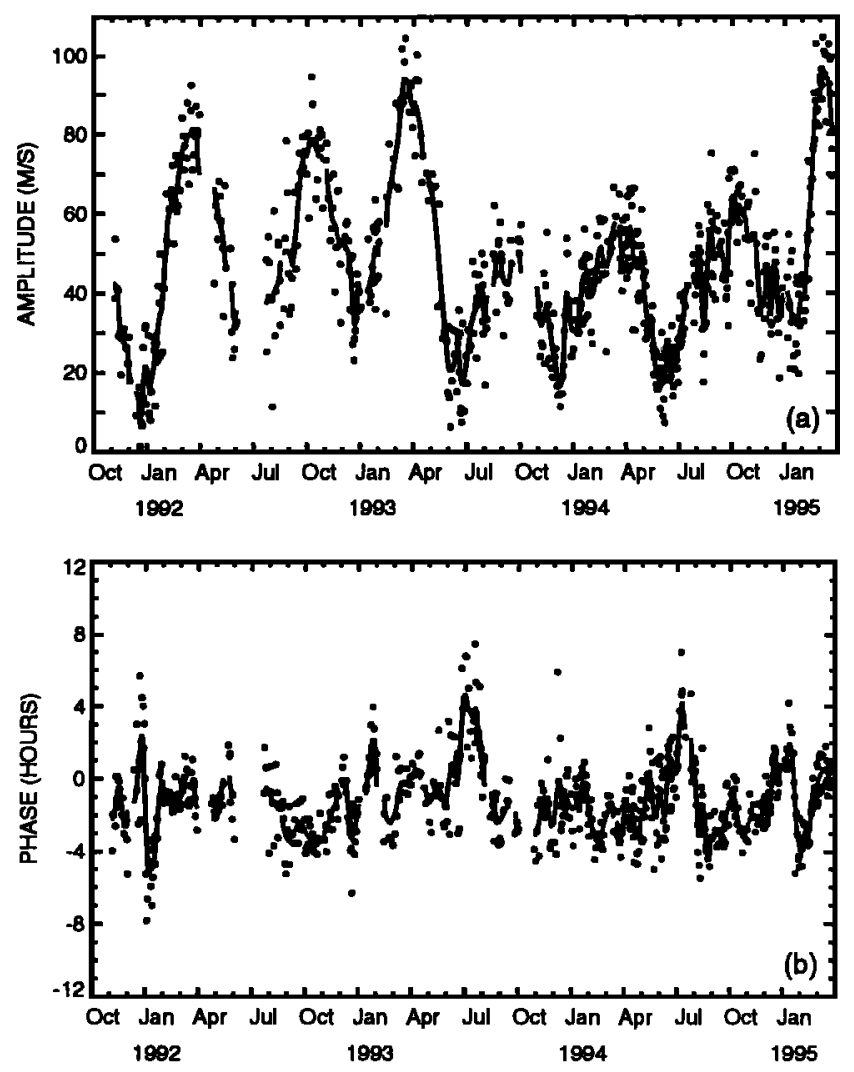

Figure 1. Daily estimates of the $(1,1)$ diurnal component of the meridional wind obtained from HRDI data for an altitude of 95 $\mathrm{km}$ and a latitude of $20^{\circ}$. The upper panel (a) shows the derived amplitudes and the lower panel (b) the phases, defined as the local time of the maximum positive (northward) wind at a latitude of $20^{\circ} \mathrm{N}$. The solid line is a 10-day running average, which serves to highlight the long-term variations.
However, earlier in the mission the nighttime wind mode was performed much less frequently than the daytime measurements so that the nighttime local time bins were typically not well filled. Also, tests indicate that the use of $95-\mathrm{km}$ nighttime data makes very little difference to the tidal retrievals. If the analysis were carried out only in the time domain, then biases could arise from semidiurnal tide contamination in regions where the semidiurnal amplitude was comparable to that of the diurnal tide. Since the fit is performed in three dimensions, time, latitude and altitude, given the different phasing, latitude structure and vertical wavelengths of the semidiurnal tide relative to the $(1,1)$ component, any contamination of the $(1,1)$ retrieval will be negligible.

The daily $(1,1)$ amplitudes and phases corresponding to a latitude of $20^{\circ}$ and an altitude of $95 \mathrm{~km}$ are presented in Figure 1 for the period from November 1991 to February 1995. In order to enhance the long-term variations, a 10-day running mean is also included in the figure. The results exhibit significant variability from day to day, but also display a clear long-term modulation with amplitude maxima near the equinoxes and minima near the solstices. This semiannual variation in the diurnal tidal amplitude has been detected by ground-based radars at Adelaide and at Kyoto [Vincent et al., 1989]. An even longer-term modulation of the diurnal tide is also evident in Figure 1. In particular, the maxima are in the range $90-110 \mathrm{~ms}^{-1}$ prior to the middle of 1993 , but for the September equinox of 1993 the peak amplitude is only about $60 \mathrm{~ms}^{-1}$. By September 1994 the maximum amplitude, although still relatively weak, has increased to $\sim 75 \mathrm{~ms}^{-1}$, and at the end of the period (February 1995) there is evidence of a further enhancement of the equinox maximum. Vincent et al. [1988] found a similar degree of interannual variability at both the Adelaide and Kyoto radar stations, observing amplitudes in 1985 that were some $40-60 \%$ greater than those measured in 1984 . Because the results illustrated in Figure 1 are representative of diurnal tidal interannual variability over a fairly broad range of latitudes, the present study suggests that the phenomenon is truly a global one. Any consistent long-term modulation of the phases shown in Figure 1 are much less clear than for the amplitudes. However, there is a suggestion that the phases move to later local times during the solstice periods.

\section{Comparisons With the GSWM}

GSWM simulations of the diurnal tide are presently limited in the resolution of seasonal variability by the availability of only four monthly mean tropospheric forcing rates [Groves, 1982b], corresponding to January, April, July, and October. There is currently no capability to simulate interannual variability. Consequently, for comparison with the model, the more than 3 years of HRDI amplitudes and phases were combined into twelve monthly averages (e.g., January 1992, January 1993, January 1994 , and January 1995 were used to produce the January values) yielding an observational mean seasonal climatology. In Figure 2 the comparison of the observed and simulated seasonal variations are presented for a latitude of $20^{\circ}$ and an altitude of $100 \mathrm{~km}$. The amplitudes are in good agreement for June and July, but for April and October the model indicates a much weaker tide than is observed. Clearly, HRDI detects a much larger seasonal variation in amplitude than is predicted by the GSWM. It should be noted that although the HRDI climatology was generated by combining different years, the degree of seasonal variability is rather similar throughout (Figure 1). Unlike the results of the HRDI analysis, which is designed to extract the $(1,1)$ component of the diurnal tide, the GSWM tropospheric forcing includes contributions from the $(1,-2),(1,1),(1,-4),(1,-1),(1,3)$, and $(1,2)$ modes [Groves, $1982 \mathrm{~b}]$. The new strato-mesospheric forcing in GSWM does not invoke the approximations associated with modal decomposition so that the model cannot be employed to generate only the $(1,1)$ component of the tide. This will cause some discrepancy between the predictions and the observations (e.g., the model shows 

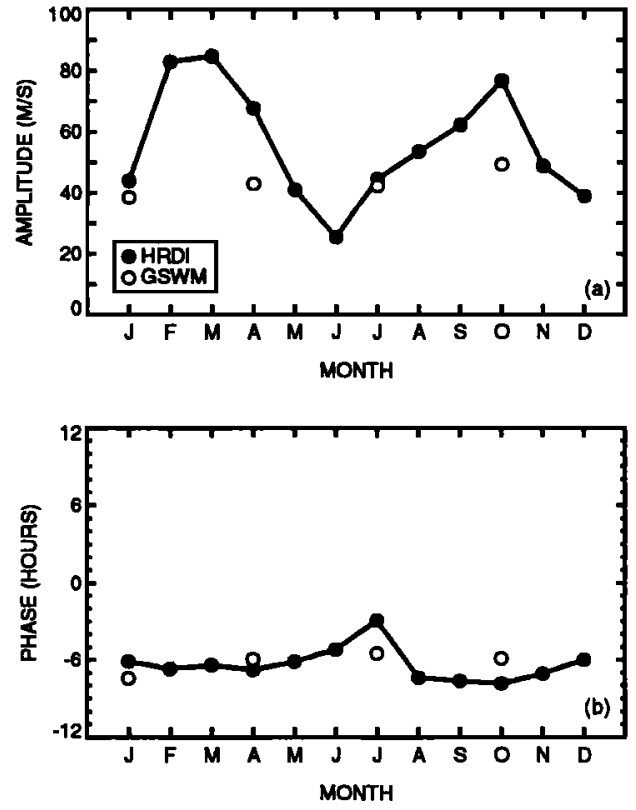

Figure 2. Comparison of HRDI seasonal climatology of the diurnal tide in the meridional wind with GSWM results for a latitude of $20^{\circ}$ and an altitude of $100 \mathrm{~km}$. The upper panel (a) shows the amplitudes and the lower panel (b) the phases.

small but significant interhemispheric asymmetry in the tide). However, since the $(1,1)$ component is also dominant in the model, this does not explain the predicted very weak seasonal variation in the amplitude relative to the HRDI data. The model phase shows a similarly weak seasonal cycle, with the largest discrepancy of about 3 hours occurring in July.

In Figure 3 the HRDI and model amplitudes and phases are shown as a function of altitude for the months of April and July. For April, the HRDI amplitudes are larger than predicted above $85 \mathrm{~km}$, but smaller below this altitude. In July, the model ampli-
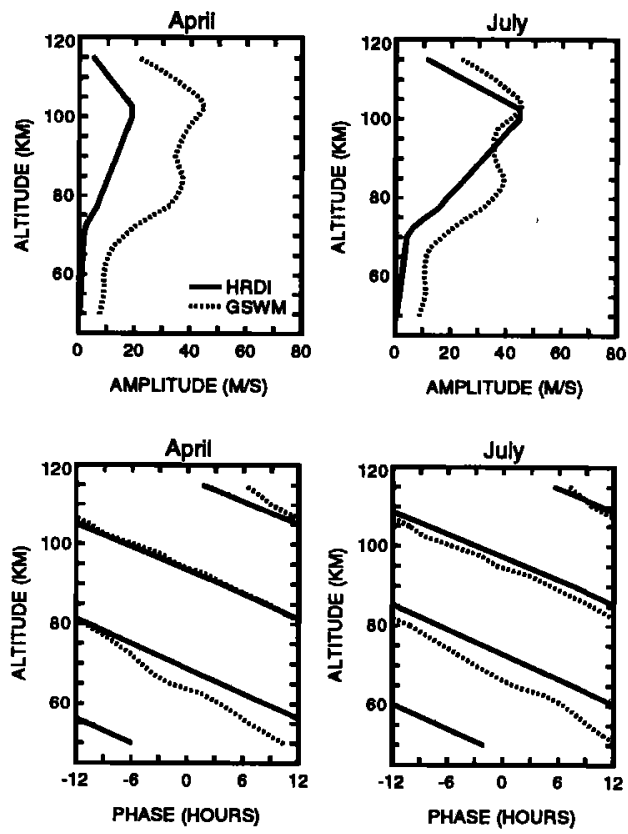

Figure 3. Comparison of HRDI meridional wind diurnal tidal amplitudes and phases with GSWM results as a function of altitude at a latitude of $20^{\circ}$ for the two months April and July. cudes are larger than observed at all altitudes except between about 90 and $105 \mathrm{~km}$, where they are comparable. In both seasonal cases the measurements and the simulations show a peak amplitude near $100 \mathrm{~km}$. The model also predicts a second peak at $85 \mathrm{~km}$, a feature which does not appear in the observations. The phases are in generally good agreement for both months, except that below $80 \mathrm{~km}$ the model indicates a vertical wavelength of approximately $30 \mathrm{~km}$, whereas the HRDI value below $80 \mathrm{~km}$ has been found to be about $25 \mathrm{~km}$ for the entire data set.

\section{Discussion}

Earlier simulations of the diurnal tide, such as those of Vial [1989], ascribed an important role to variable mean winds. However, it should be noted that previous numerical studies have not included seasonal variability in parameterizations of eddy dissipation, and the GSWM is the first tidal model to include the effects of gravity wave stress. Above $20 \mathrm{~km}$ the winds poleward of latitude $10^{\circ}$ in the GSWM calculations are obtained from the MSISE90 [Hedin, 1991] zonal mean pressure gradients assuming geostrophic balance. These values are then interpolated across the equatorial region. A second series of GSWM calculations including dissipation and a modified background wind field were carried out to test the sensitivity of GSWM predictions to more realistic mean winds. Above $80 \mathrm{~km}$, the GSWM background was specified by the empirical zonal mean zonal wind model of Portnyagin and Solov'eva [1992a, b]. Differences between this series and the results illustrated in Figure 2 were within $5 \mathrm{~ms}^{-1}$ during all seasons. This suggests the comparative insensitivity of the diurnal tide to changes in background MLT winds. However, some of the details of the interhemispheric asymmetries in GSWM results [Hagan et al., 1995] are sensitive to background wind conditions. Additional numerical experiments using HRDI data to further investigate the role of mean winds are planned, once the HRDI stratospheric wind product $(10-40 \mathrm{~km})$ is fully validated. At present, the employment of HRDI background zonal winds in the GSWM background is precluded by significant differences between the HRDI MLT winds and the GSWM geostrophic winds at the $50 \mathrm{~km}$ altitude of interface between the two.
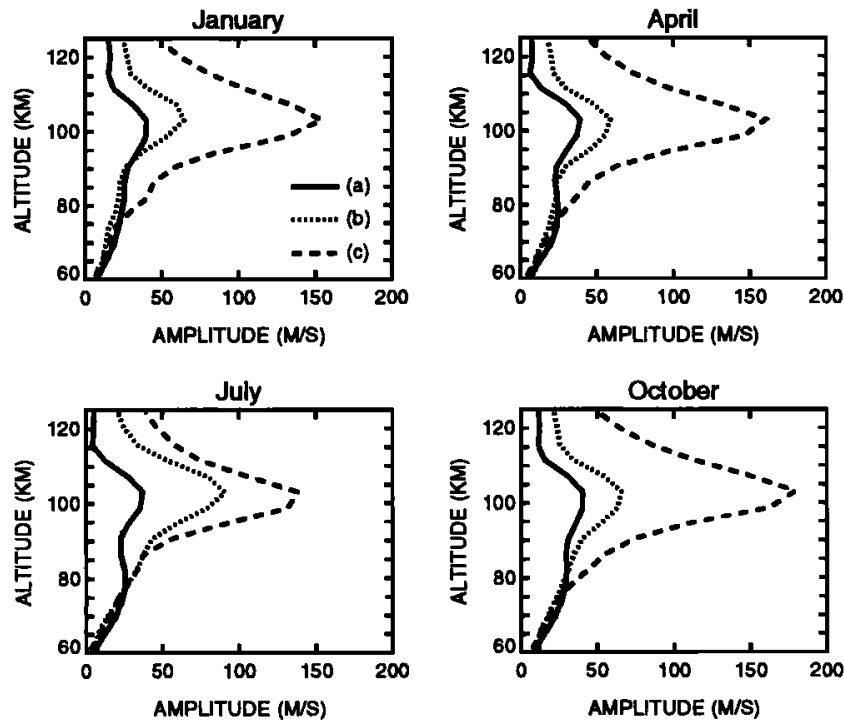

Figure 4. Amplitude of the diurnal tide in the meridional wind at $20^{\circ} \mathrm{N}$ as a function of altitude simulated by the GSWM for the four months of January, April, July, and October. The three model cases shown correspond to (a) zero zonal mean wind, (b) zero wind and no gravity wave stress, and (c) zero wind and no dissipation. 
A series of additional GSWM calculations were performed to deconvolve the comparative importance of seasonally variable thermotidal forcing and tidal dissipation in the model predictions. The meridional amplitudes at $20^{\circ} \mathrm{N}$ which result from these are presented in Figure 4. Simulations which exclude background winds but include the effects of gravity-wave dissipation in the form of eddy diffusivity (which accounts for turbulence) and effective Rayleigh friction (wave stress) are characterized by weak seasonal variability [case (a) in Figure 4]. The peak meridional amplitudes are remarkably similar to, though slightly smaller than, those illustrated in Figures 2 and 3. Specifically, they are of comparable magnitude during January, April, and July, and comparatively larger in October.

Case (b) in Figure 4 depicts the results with zero winds and no gravity-wave stress, but including eddy diffusivity effects. In this case, the largest amplitude occurs in July and the smallest in April, which is in complete disagreement with the HRDI observations (Figure 3). Although Garcia and Solomon [1985] concluded that eddy diffusion is larger during solstice (implying a smaller tide) than for equinox, close examination of their results indicates that this is not true at all latitudes. Specifically, over the latitude range $-30^{\circ}$ to $30^{\circ}$, which is critically important for the diurnal tide, the reverse is true and the GSWM case (b) tidal predictions are consistent with this result.

In the absence of background winds, gravity-wave stress and eddy diffusivity [case (c) in Figure 4], GSWM meridional amplitudes peak in excess of $100 \mathrm{~ms}^{-1}$ near $102-\mathrm{km}$ altitude and $20^{\circ}$ latitude. They are weakest in July, comparable during January and April, and strongest during October with July/October differences approaching $40 \%$. These results suggest that there is significant seasonal variability in GSWM thermotidal forcing and that these signatures are not directly manifested in the model meridional amplitude predictions when background winds and dissipative processes are included (Figure 2). Thus, the weak seasonal variability of the GSWM meridional wind amplitude is largely attributable to the tidal dissipation employed in the model

These results point to the need for improvements to the parameterization of tidal dissipation in GSWM. Several investigations are underway but a simple tuning of the eddy diffusivities to match the GSWM diurnal tide to HRDI observations is not justified. First, such a scheme would produce an unrealistically large semidiurnal tide. Further, effects of gravity-wave stress on the diurnal tide are currently invariant with season in GSWM and will be further developed to include seasonal variability. Investigating the seasonal variability of gravity-wave drag effects is promising, since these only affect the diurnal component [e.g., Vial and Forbes, 1989]. Finally, there is a body of ground-based evidence to be considered. For example, Nakamura et al. [1993] observed larger gravity-wave wind variances under solstice conditions than during the equinoxes, which is consistent with the observed semiannual variation in the amplitude of the diurnal tide. Further evaluation of, and extension to, the current parameterization of tropospheric thermotidal forcing in the GSWM [Groves, 1982b] is also planned, since the current scheme is based upon 3-month averages of humidity measurements which are more than 30 years old.

\section{Summary}

The $(1,1)$ diurnal tidal amplitudes have been derived for the more than 3-year span of the HRDI meridional wind data set. This has revealed a strong interannual variability in the diurnal tide, and in particular the amplitudes obtained prior to June 1993 were substantially larger than those observed subsequently. Regular seasonal changes are also detected, which are much stronger than predicted by the current version of the GSWM. The results of the comparison, together with numerical experiments, highlight important and unresolved questions regarding the mechanisms contributing to the dynamics of the MLT region.
Acknowledgments. This work is sponsored by NASA through Contract NAS 5-27751. The efforts of M. E. Hagan are supported by the NSF CEDAR program and NASA grant S-41353-F. The authors would like to thank S. E. Palo for useful comments on the manuscript.

\section{References}

Burrage, M. D., et al. Observations of the $\mathrm{O}_{2}$ atmospheric band nightglow by the High Resolution Doppler Imager, J. Geophys. Res., 99, 15017 $15023,1994$.

Chapman, S., and R. S. Lindzen, Atmospheric Tides, D. Reidel, Hingham, Mass., 1970.

Fesen, C. G., R. E. Dickinson, R. G. Roble, Simulation of the thermospheric tides at equinox with the National Center for Atmospheric Research thermospheric general circulation model, $J$. Geophys. Res., 91, 4471-4489, 1986.

Forbes, J. M., Atmospheric tides 1. Model description and results for the solar diurnal component, J. Geophys. Res., 87, 5222-5240, 1982.

Forbes, J. M., and F. Vial, Monthly simulations of the solar semi-diurnal tide in the mesosphere and lower thermosphere, J. Atmos. Terr. Phys., $51,649-661,1989$.

Garcia, R. R., and S. Solomon, The effect of breaking gravity waves on the dynamics and chemical composition of the mesosphere and lower thermosphere, J. Geophys. Res., 90, 3850-3868, 1985.

Groves, G. V., Hough components of ozone heating, J. Atmos. Terr. Phys., 44, 111-121, 1982a.

Groves, G. V., Hough components of water vapour heating, J. Atmos. Terr. Phys., 44, 281-290, $1982 \mathrm{~b}$.

Hagan, M. E., J. M. Forbes, and F. Vial, A numerical investigation of the propagation of the quasi 2-day wave into the lower thermosphere, $J$. Geophy. Res., 98, 23193-23205, 1993.

Hagan, M. E., J. M. Forbes, and F. Vial, On modelling migrating solar tides, Geophys. Res. Lett., 22, 893-896, 1995.

Hays, P. B., et al., The High-Resolution Doppler Imager on the Upper Atmosphere Research Satellite, J. Geophy. Res., 98, 10713-10723, 1993.

Hays, P. B., et al., Observations of the diurnal tide from space, J. Atmos. Sci., 51, 3077-3093, 1994.

Hedin, A. E., et al. Revised global model of thermosphere winds using satellite and ground-based observations, J. Geophys. Res., 96, 76577688, 1991.

Manson, A. H., et al., Climatologies of semi-diurnal and diurnal tides in the middle atmosphere $(70-110 \mathrm{~km})$ at middle latitudes $\left(40^{\circ}-55^{\circ}\right), J$. Atmos. Terr. Phys., 51, 579-593, 1989.

Nakamura, T., et al., Comparative observations of short-period gravity waves (10-100 $\mathrm{min}$ ) in the mesosphere in 1989 by Saskatoon MF radar $\left(52^{\circ} \mathrm{N}\right)$, Canada and the MU radar ( $\left.35^{\circ} \mathrm{N}\right)$, Japan, Radio Sci, 28, 729$746,1993$.

Portnyagin, Y. I., and T. V. Solov'eva, An empirical model of the meridional wind in the mesopause-lower thermosphere, Part 1 . A mean monthly empirical model, Russian J. Met. and Hydr., 10, 28-35, 1992a.

Portnyagin, Y. I., and T. V. Solov'eva, An empirical model of the meridional wind in the mesopause/lower thermosphere, Part 2. Heightlatitude features of basic components of meridional wind seasonal variations, Russian J. Met. and Hydr., 11, 29-36, $1992 \mathrm{~b}$.

Vial, F., Tides in the middle atmosphere, J. Atmos. Terr. Phys., 51, 3-17, 1989.

Vial, F., and J. M. Forbes, Recent progress in tidal modelling, J. Atmos. Terr. Phys., 51, 663-671, 1989.

Vincent, R. A., T. Tsuda, and S. Kato, A comparative study of mesospheric solar tides observed at Adelaide and Kyoto, J. Geophy. Res., 93, 699-708, 1988.

Vincent, R. A., T. Tsuda, and S. Kato, Asymmetries in mesospheric tidal structure, J. Atmos. Terr. Phys., 51, 609-616, 1989.

M. D. Burrage, P. B. Hays, and W. R. Skinner, Space Physics Research Laboratory, University of Michigan, Ann Arbor, MI 48109. (email: burrage@sprlc.sprl.umich.edu; hays@sprlj.sprl.umich.edu; wskinner@umich.edu)

M. E. Hagan, National Center for Atmospheric Research, High Altitude Observatory, Boulder, CO 80307. (e-mail: hagan@ tatooine.hao.ucar.edu)

D. L. Wu, Jet Propulsion Laboratory, California Institute of Technology, Pasadena, CA 91 109. (e-mail: dwu@camel.jpl.nasa.gov)

(Received May 15, 1995; revsied August 1, 1995; accepted August 15, 1995.) 